\title{
Hybrid Particle-based Full-band Analysis of Ultra-small MOS
}

\author{
S. J. WIGGER ${ }^{\mathrm{a}, *}$, S. M. GOODNICK ${ }^{\mathrm{a}}$ and M. SARANITI ${ }^{\mathrm{b}}$ \\ ${ }^{a}$ Department of Electrical Engineering and Center for Solid State Electronics Research Arizona State University, \\ Tempe, AZ 85287-5706; ' Department of Electrical and Computer Engineering, \\ Illinois Institute of Technology, Chicago, IL 60616-3793
}

\begin{abstract}
We report on the 2D and 3D modeling of ultra-small MOS structures using a newly developed full-band device simulator. The simulation tool is based on a novel approach, featuring a hybrid Ensemble Monte Carlo (EMC)-Cellular Automata (CA) simulation engine. In this hybrid approach charge transport is simulated using the $\mathrm{CA}$ in regions of momentum space where most scattering events occur and the EMC elsewhere, thus optimizing the trade-off between the fast, but memory consuming CA method and the slower EMC method. To account for the spatial distribution of the electric field and charge concentration, the hybrid EMC/CA simulator is self-consistently coupled with a 2D and 3D multi-grid Poisson solver. The solver is then used to simulate the performance of a $40 \mathrm{~nm}$ gate length n-MOSFET structure.
\end{abstract}

Keywords: Monte Carlo; Cellular Automata; Device modeling; Multi-grid

\section{INTRODUCTION}

The general trend in the electronics industry towards more highly integrated circuits, is driving the size of semiconductor devices into the deep sub-micron regime. Presently, commercial MOSFET structures are nearing gate lengths of $0.1 \mu \mathrm{m}$, and experimental devices with gate lengths on the order of $40 \mathrm{~nm}$ structures have been achieved [1].

In order to simulate these deep sub-micron devices, modeling tools must account for the highly non-linear nature of the charge transport. The electronic structure and phonon spectra need to be represented with a full-band energy momentum relation and full 3-D carrier motion needs to be included.

The EMC method has long demonstrated success in simulating carrier transport in semiconductor devices [2,3]. Unfortunately, this technique can be computational intensive, limiting its application, particularly when a full-band representation of the electronic structure is implemented. The CA method was developed to reduce this computational burden $[4,5]$, and although faster, it can often require an unrealistic amount of memory. A new hybrid EMC/CA method has been proposed [6], which optimizes the trade-off between memory and speed, while retaining accuracy.

*Corresponding author. e-mail: shela.wigger@asu.edu 
The hybrid dynamics simulator is self-consistently coupled with both a 2D and 3D multi-grid Poisson solver in order to resolve the spatial distribution of the electric field. The simulator is then used to model a $40 \mathrm{~nm}$ gate length nMOSFET, with an effective channel length of $27 \mathrm{~nm}$ corresponding to devices which were fabricated by Ono et al. [1].

\section{HYBRID EMC/CA}

The first Brillouin zone (BZ) is discretized using a non-uniform mesh for both electrons and holes, and the band structure is calculated using the empirical pseudopotential method [7], with spin orbit interaction included. The transition tables are generated using non-polar phonons, ionized impurity scattering, and impact ionization as in Fischetti and Laux [3]. In the CA formalism, the momentum dependent scattering probability is stored for every initial state to every final state, satisfying energy conservation. This leads to a considerable simplification in the final state selection after scattering, which is typically a time consuming process in full-band EMC simulations. The nature of the CA algorithm makes any correction to the energy after scattering impossible, therefore a trade-off exists between the energy resolution in momentum space, and the size of the transition tables.

The hybrid implementation allows for the use of the fast, but memory consuming CA in the most active regions of momentum space, while using the EMC elsewhere, as shown in Figure 1. The small EMC scattering tables are inserted in regions where the relative number of scattering events is small, such as in the high energy portions of the $\mathrm{BZ}$, and also in regions of momentum space where a higher degree of accuracy is necessary, such as close to the minima of the first conduction band and the maxima of the valence band. Since the number of scattering events will be low, the EMC will not significantly affect the overall performance of the simulator, but it will help to reduce the

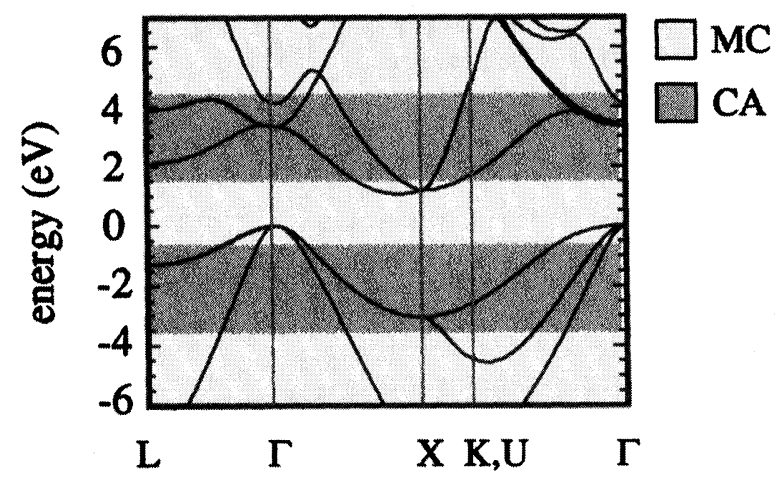

FIGURE 1 Representation of regions of Brillouin zone where the Cellular Automata and the Monte Carlo are implemented.

amount of required memory so that the CA can more accurately select an energy conservation final state.

The equivalence between the hybrid EMC/CA and a traditional full-band EMC is demonstrated in Figure 2 for the electron number at two different fields for Si. In spite of the sensitivity of the energy distribution on the transport model, the agreement of the two approaches is quite good. A difference is noticeable in the high-energy tail of each curve. The higher values given by the hybrid approach are due to its smaller energy resolution in those regions of $\mathrm{BZ}$ that are far from the minima of the conduction band. This smaller resolution produces a spurious diffusion (i.e., a numerical heating) of electrons toward higher energies

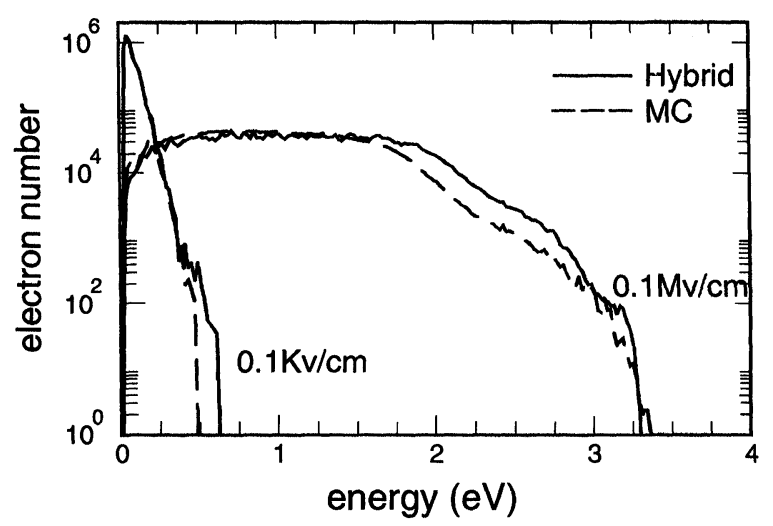

FIGURE 2 Electron number as computed for the Monte Carlo and the hybrid method. 


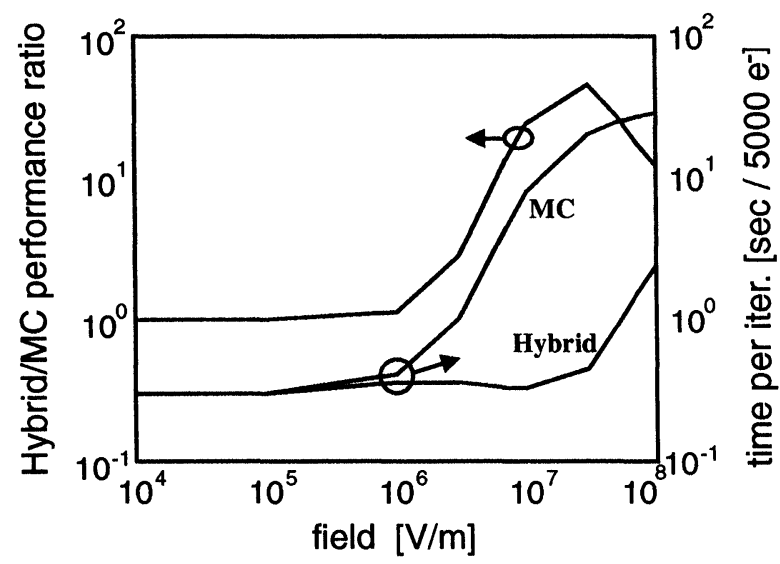

FIGURE 3 Comparison of performance between the hybrid and the MC algorithm. The hybrid can demonstrate a speed-up of 45 times.

regions. The effect is not present in the section above $4 \mathrm{eV}$ of the distribution calculated at $0.1 \mathrm{MV} / \mathrm{cm}$, because the numerical heating is compensated by the cooling effect of impact ionization. The small discrepancy between the EMC and the hybrid result can be easily reduced by grid refinement.

A comparison between the execution time of the hybrid and an efficient EMC algorithm is shown in Figure 3 in order to demonstrate the relative speed-up of the hybrid simulator. The simulation was run for 5000 electrons, on a processor running at $400 \mathrm{MHz}$. In the low field regeme, the hybrid is dominated by the EMC method, since carriers have low energies, but as the field increases past $10^{6} \mathrm{~V} / \mathrm{m}$, the performance of the hybrid is greatly improved, due to the CA scattering selection. As seen in Figure 3, the hybrid approach can give a speed-up of as much as 45 times. In the case of electrons in $\mathrm{Si}$, the memory necessary for a full EMC simulation is 130 Mbytes, while a CA simulation requires 1.2 Gbytes.

\section{DEVICE SIMULATION}

The hybrid EMC/CA is self consistently coupled with a $2 \mathrm{D}$ and $3 \mathrm{D}$ multi-grid Poisson solver to account for the spatial distribution of the electric field. In order to demonstrate the applicability of the hybrid approach, simulations are run for a $40 \mathrm{~nm}$ gate length n-MOSFET, based on the fabricated device of [1], including the non-uniform impurity profile of the source drain contact regions.

The schematic layout of this ultra-small structure is shown in Figure 4a. The p-doped substrate has a concentration of $\mathrm{N}_{\mathrm{A}}=1 \times 10^{18} \mathrm{~cm}^{-3}$, and the source and drain doping corresponds to an ion implanted Gaussian doping profile with a peak value of $\mathrm{N}_{\mathrm{D}}=1 \times 10^{19} \mathrm{~cm}^{-3}$, while the highly doped regions at the ends of the channel have a peak doping of $\mathrm{N}_{\mathrm{D}}=1 \times 10^{21} \mathrm{~cm}^{-3}$. The cross section of the doping profile along the drain junction is also shown in Figure 4a. Due to the side diffusion of the source drain implants, the effective channel length is $27 \mathrm{~nm}$.

The $40 \mathrm{~nm}$ device is simulated in 2D using an inhomogeneous, rectangular grid with $129 \times 65$ nodes. 32,000 super-particles are used to represent the electrons and 34,000 are used for the holes. In $3 \mathrm{D}$ the mesh is $129 \times 65 \times 9$, and therefore requires a higher number of simulated particles.

Due to spatial fluctuations in the carrier distribution, a trade-off exists between the number of simulated particles and the Poisson time step length. Therefore, a very small time step is used for updating the solution of Poissons equation, here taken to be $0.2 \mathrm{fs}$, which also corresponds to the free flight time step.

Figure $4 \mathrm{~b}$ shows the calculated $\mathrm{I}-\mathrm{V}$ curves obtained from the 2D simulator. Identical results between the 2D and 3D simulators are obtained assuming a uniform structure the third dimension. However, the execution speed is more than an order of magnitude slower for the 3D simulation, which precludes the generation of extensive $\mathrm{I}-\mathrm{V}$ characteristics on the workstation used for the full band simulations. The calculated $\mathrm{I}-\mathrm{V}$ curves show significantly more output conductance and higher current than the Toshiba device [1]. The inclusion of additional scattering mechanisms may account for some of the discrepancy. Conversely, 


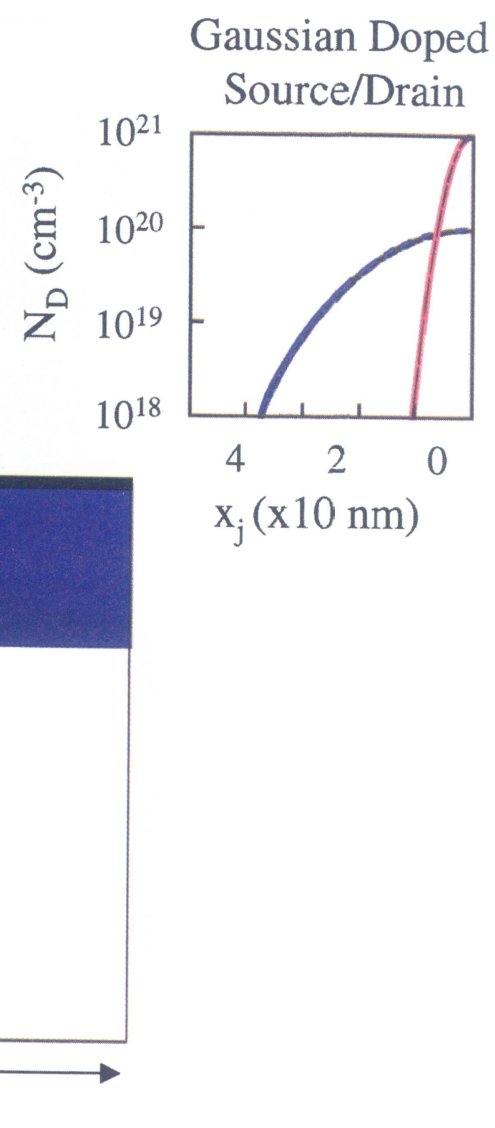

(a)

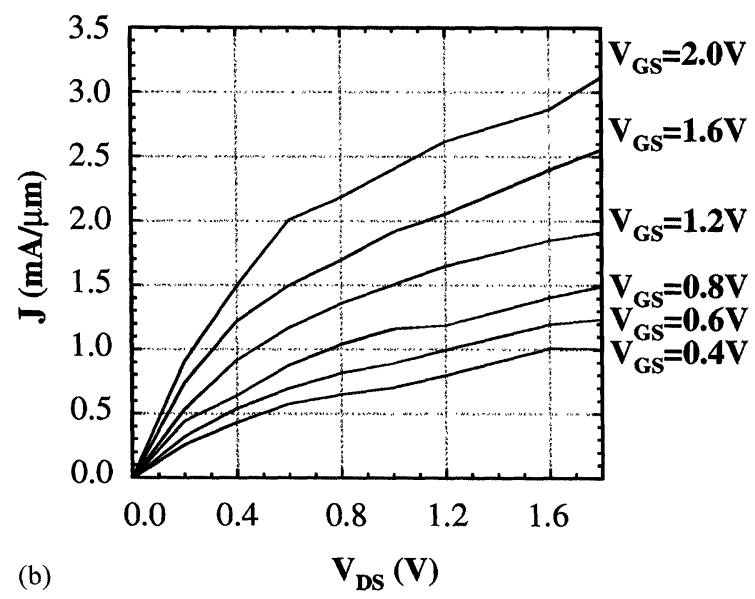

FIGURE 4 (a) Schematic layout of a $40 \mathrm{~nm}$ n-MOSFET, corresponding to a fabrication process described in [1]. The oxide thickness is $3 \mathrm{~nm}$, and the bulk is doped $\mathrm{N}_{\mathrm{A}}=1 \times 10^{18} \mathrm{~cm}^{-3}$. The source and drain regions are doped using a Gaussian profile, with a peak concentration of $N_{D}=1 \times 10^{20} \mathrm{~cm}^{-3}$, higher doped regions are located on each side of the channel and have a peak concentration of $N_{D}=1 \times 10^{21} \mathrm{~cm}^{-3}$. The effective channel length is $27 \mathrm{~nm}$. (b) $\mathrm{I}-\mathrm{V}$ characteristics corresponding to the $2 \mathrm{D}$ simulation. (See Color Plate IV). 
a longer effective channel length than that reported experimentally also may lead to a closer agreement.

\section{FUTURE WORK}

The hybrid algorithm is shown to be a more computationally efficient particle-based method over the tradition EMC and CA methods. As a natural evolution of this approach, a parallel version of the alogorithm is currently being implemented.

The repeated solution of the Poisson equation dominates the computation time in $3 \mathrm{D}$, due to the large number of real space grid cells needed for a 3D mesh, and to the larger number of particles necessary for the simulation of electrons and holes. Parallelization of the multi-grid Poisson solver is currently being implemented, in order to overcome this computational burden.

\section{Acknowledgements}

The authors would like to acknowledge the support of this research by the National Science
Foundation Grant ECS-9976484. The authors would also like to thank D. Vasileska for her assistance.

\section{References}

[1] Ono, M., Saito, M., Yoshitomi, T., Fiegna, C., Ohguro, T. and Iwai, H. (1995). "A $40 \mathrm{~nm}$ Gate Length n-MOSFET", IEEE Trans. Electron Dev., 42, 1822-30.

[2] Jacoboni, C. and Reggiani, L. (1983). "The Monte Carlo method for solution of charge transport in semiconductors with applications to covalent materials", Rev. Mod. Phys., 55, 645.

[3] Fischetti, M. V. and Laux, S. E. (1988). "Monte Carlo analysis of electron transport in small semiconductor devices including band-structure and space-charge effects", Phys. Rev. B., 38, 9721-45.

[4] Kometer, K., Zandler, G. and Vogl, P. (1992). "Lattice-gas cellular-automaton method for semiclassical transport in semiconductors", Phys. Rev. B., 46, 1382.

[5] Saraniti, M., Wigger, S. J. and Goodnick, S. M. (1999). "Full-Band cellular automata for modeling transport in sub-micron devices", Proc. of MSM99, S. Juan (PR), pp. $380-383$.

[6] Saraniti, M. and Goodnick, S. M., "Hybrid Full-Band Cellular Automaton/Monte Carlo Approach for Fast Simulation of Semiconductor Devices", Accepted for publication in IEEE Trans Electron Dev.

[7] Chelikowsky, J. R. and Cohen, M. L. (1976). "Nonlocal pseudopotential calculations for the electronic structure of eleven diamond and zinc-blend semiconductors", Phys. Rev. B., 14, 556-582. 

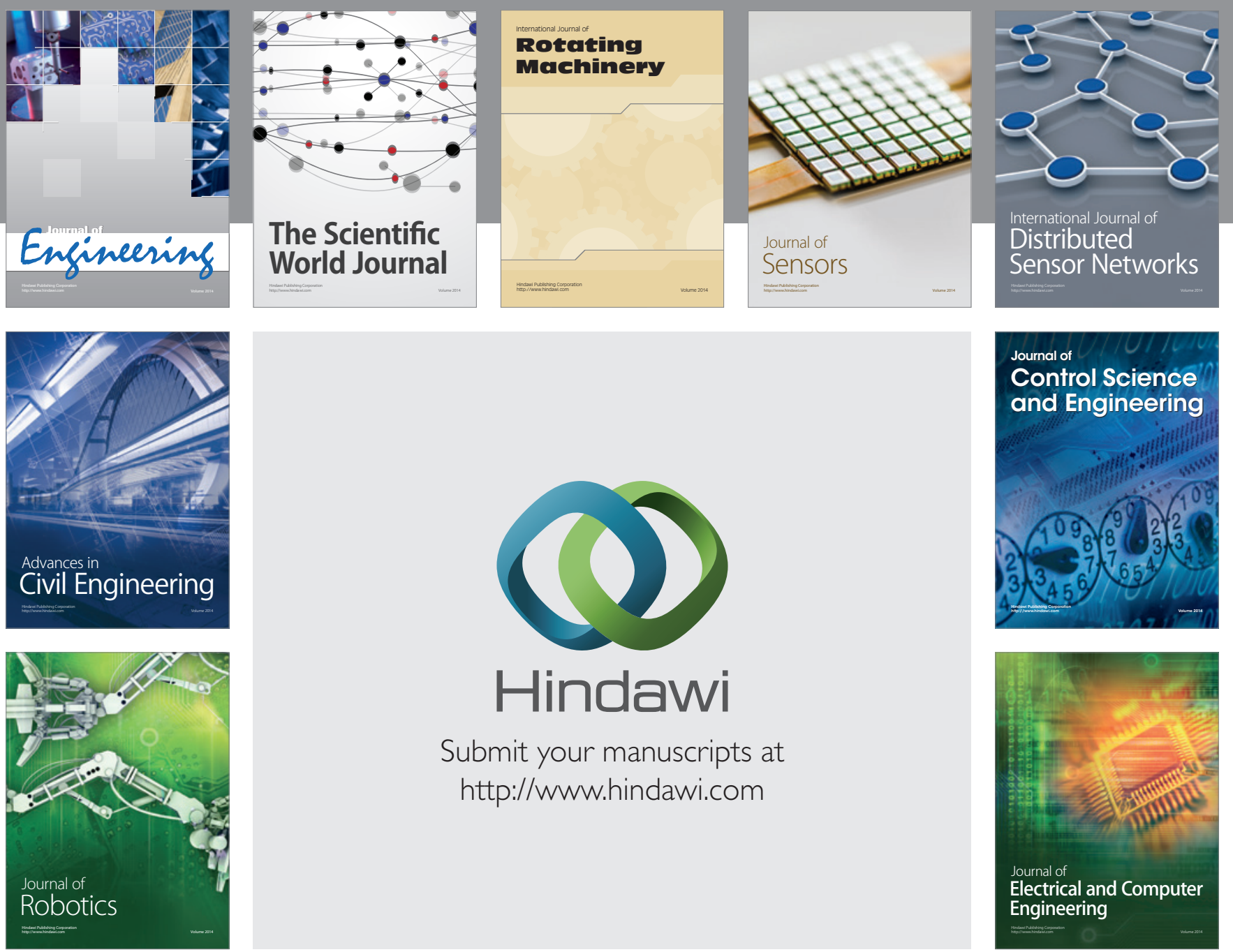

Submit your manuscripts at

http://www.hindawi.com
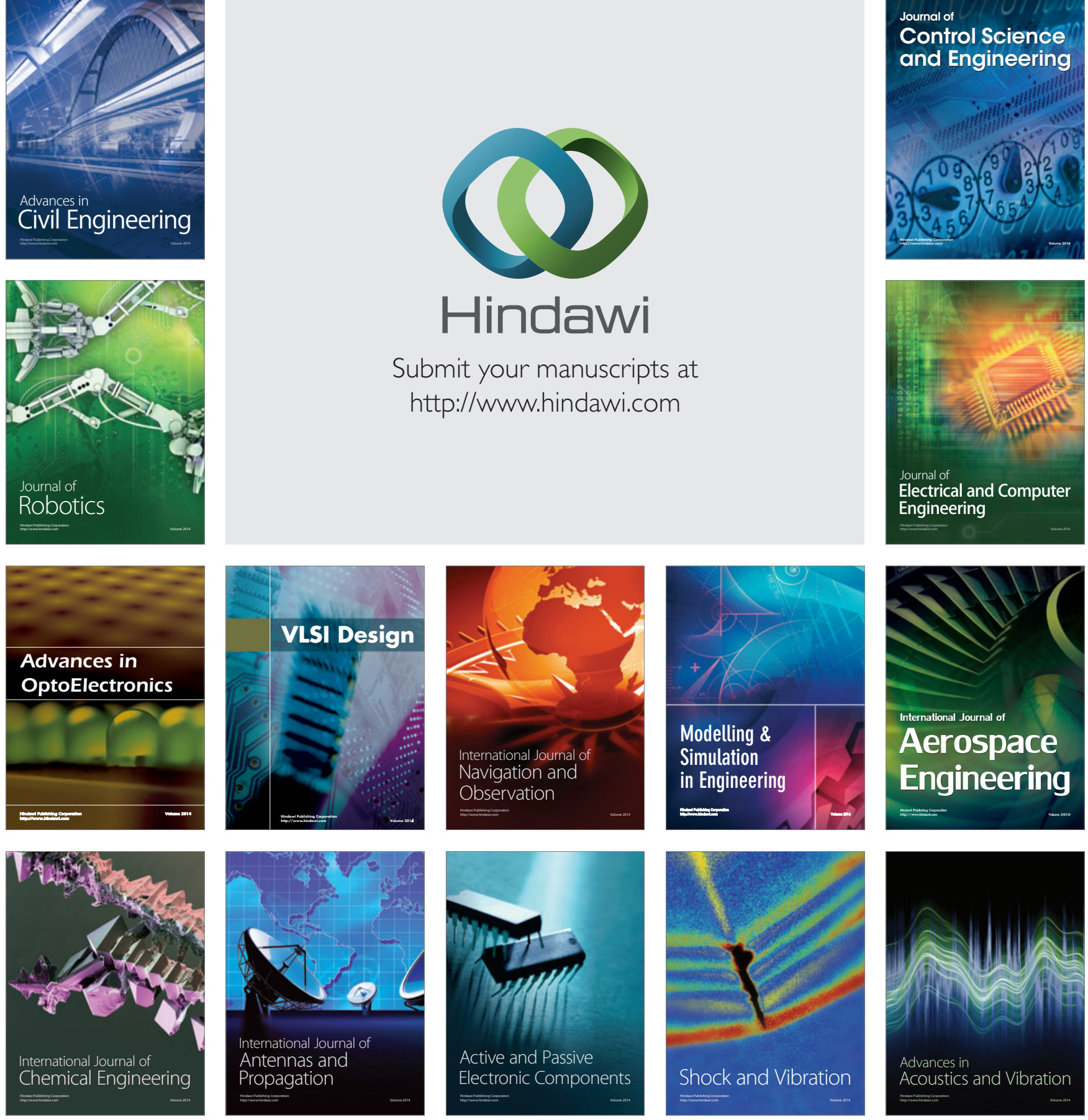